\title{
Non-Calcified Coronary Artery Plaque Characterization by Dual-Energy Computed Tomography
}

\author{
Rezvan Ravanfar Haghighi, ${ }^{1,}$ Sabyasachi Chatterjee, ${ }^{2}$ Vardahan.C. Vani, ${ }^{3}$ Pratik Kumar, ${ }^{4}$ Milo Tabin, ${ }^{5}$ \\ Ruma Ray, ${ }^{6}$ Reza Jalli, ${ }^{1}$ Sepide Sefidbakht, ${ }^{1}$ Fariba Zarei, ${ }^{1}$ Ali Reza Shakibafard, ${ }^{1}$ Somayeh Gholami \\ Bardeji, ${ }^{1}$ Mahdi Dodangeh, ${ }^{1}$ and Bijan Bijan ${ }^{1,7}$ \\ ${ }^{1}$ Medical Imaging Research Center, Shiraz University of Medical Sciences, Shiraz, Iran \\ ${ }^{2}$ BGVS Chemical Engineering Building (Old), Indian Institute of Science, Bangalore, India \\ Department of Instrumentation and Applied Physics, Indian Institute of Science, Bangalore, India \\ ${ }^{4}$ Medical Physics Unit, IRCH, All India Institute of Medical Sciences, Ansari Nagar, New Delhi, India \\ ${ }^{5}$ Department of Forensic Medicine, All India Institute of Medical Sciences, New Delhi, India \\ ${ }^{6}$ Department of Pathology, All India Institute of Medical Sciences, New Delhi, India \\ Department of Radiology, Sutter Medical Group/University of California Davis, Sacramento, California, USA \\ Corresponding author: Rezvan Ravanfar Haghighi, Medical Imaging Research Center, Shiraz University of Medical Sciences, Shiraz 7134845794, Iran. Tel: +98-7136281464, Fax: \\ +98-7136281506, E-mail: sravanfarr@gmail.com
}

Received 2017 April 05; Revised 2017 October 04; Accepted 2017 October 27.

\begin{abstract}
Background: Inversion of dual-energy computed tomography (DECT) data for obtaining the electron density and effective atomic number of substances has been a work in progress for the past forty years. It has been the practice to characterize the material in terms of Hounsfield Unit (HU) values obtained by two different energies.

objectives: Since HU values are equipment-dependent quantities, it is necessary to develop a method that characterizes the substance in terms of certain physical quantities that are equipment independent.

Materials and Methods: The process that we adopt is to find a calibration method by which all equipment-dependent quantities are eliminated and we directly deal with quantities that are representative of the sample, namely its electron density and effective atomic number. We collect the DECT data from 21 samples of non-calcified coronary artery plaques in human cadavers.

Results: With our standardized inversion method, we have obtained the electron density and effective atomic number of these samples. With physical models of lipids and proteins, it becomes possible to conclude that non-calcified plaque samples can have calcium dispersed in the lipid part of the plaque in trace amounts that cannot be observed by light based microscopy or by CT images alone.

Conclusion: This characterization, may give a new insight in characterization of non-calcified coronary artery plaque and in medical diagnostics.
\end{abstract}

Keywords: Dual-Energy Computed Tomography, Non-Calcific Plaque, Plaque Characterization, Coronary Artery Disease

\section{Background}

Coronary artery disease (CAD) is the presentation of coronary atherosclerosis, which causes luminal narrowing of the coronary arteries by accumulation of lipid rich, fibrous, or calcified plaques to the vascular wall (1). Atherosclerotic plaque may be characterized as (1) lipid plaques with extracellular lipid with a fibrous cap, (2) fibrous plaque, and (3) calcified plaques and complex thrombotic lesions subsequent to rupture. Fibrous plaques, calcified plaques and complex lesions may cause substantial stenosis of the coronary arteries, leading to territorial ischemia and dysfunction of the affected myocardium (2). By contrast, lipid plaques, which are known to bear the highest risk of causing future cardiovascular events are usually non-stenotic (1). They may rupture and form a thrombotic occlusion, resulting in immediate or acute coronary events. Most patients with acute coronary syndromes who present with unstable angina have the risk of acute myocardial infarction, and sudden coronary death. Therefore, in acute coronary syndrome, the atherosclerotic plaque composition seems to be a critical factor for determining the risk of rupture and subsequent thrombotic catastrophic event, rather than the degree of vascular stenosis (3). Assessment of the coronary lumen, evaluation of the vessel wall and atherosclerotic plaques may offer very important information about the 
prognosis of such patients, with possible prediction of the response to treatment. However, detection and characterization of vulnerable plaques is a challenging task that may need invasive techniques such as coronary angiography (CA), intravascular ultrasound (IVUS) (4), optical coherent tomography (OCT) (5) and plaque thermography (6). An ideal diagnostic tool should be reliable, noninvasive and able to detect and characterize an unstable plaque which may potentially result in an acute coronary syndrome. Currently, two emerging and promising techniques, namely, computed tomography $(\mathrm{CT})(7,8)$ and magnetic resonance imaging (MRI) (9), are widely accepted by the medical imaging community as they are non-invasive and have the potential to evaluate luminal stenosis and characterize plaque components.

MRI is a useful system to evaluate cardiac morphology, cardiac function, myocardial perfusion and myocardial viability. It shows advantages over CT, due to lack of ionizing radiation and possessing an acceptable soft tissue resolution for atherosclerotic plaques. However, MRI has limited application because of the long overall examination time for coronary artery plaque characterization.

Computed tomography (CT) is perceived to have the potential of being one of the most important non-invasive diagnostic imaging modalities which can be used in coronary artery disease. It provides three dimensional map of the $x$-ray attenuation coefficient with sub-millimeter resolution, 0.33 seconds scan time and robust data collection enabling cardiac imagers to produce reformatted high resolution images in any given image plans (10).

The determination of tissue density expressed as Hounsfield Unit (HU value or CT-number) is a standard technique in $\mathrm{CT}$ and is used as a surrogate marker for tissue composition in most of the studies (11).

Most of the scientists performed plaque characterization using conventional multi-detector CT(MDCT) by using calculating the Hounsfield number (Hounsfield unit; HU) which is related to the attenuation coefficient of the material under study. In their analysis, they used the CT-number (HU value) of the desired area in the plaque region. They found that there is a substantial overlap between mean HU values of soft (lipid core) and intermediate (fibrous) plaque. It was, therefore, concluded that differentiation of "vulnerable" from stable plaques, based on their HU values may not be as reliable because non-calcified plaque components have similar chemical compositions and hence, there is considerable overlap in HU values (12).

Differences in tissue contrast may not be easily discernible at any single X-ray energy available on current clinical scanners. In 1976, Alvarez and Macovski (13) proposed dual-energy CT (DECT) as a method to further increase the contrast resolution. In this method, the same object is scanned at two different energies. While the conventional CT aims at finding the shape and size of the objects, embedded in the patient's body, DECT tries to determine the shape and size as well as the composition of the object.

It was shown by Rutherfored et al. (1976) that computed tomography provides a tool for precise measurement of linear attenuation coefficients of materials (14). These attenuation coefficients can be measured at different $\mathrm{x}$-ray energies and from such data it is possible to obtain separate measures of electron density $\left(\rho_{e}\right)$ and an effective atomic number $\left(Z_{e f f}\right)$ for the same object. This is the process of tissue characterization. They could discriminate dermoid cyst and meningioma by this method when the measurements were done at tube voltages 140 and 100 $\mathrm{kVp}$. In 1979, Chiro et al., used 140 and $90 \mathrm{kVp}$ computed tomography for brain scan. Their theoretical calculations and experimental studies indicated that dual-energy CT can provide clinically useful tissue signatures, not only for metallic deposits, calcification and bone or injected iodine, but was also helpful for the identification of different types of parenchyma tissues and body fluids (15).

In 2003, Heismann et al. showed that the mass density $\rho$ and effective atomic number $Z_{\text {eff }}$ of the absorber can be extracted by $\rho \mathrm{Z}$ projection algorithm from at least two attenuation values $\mu\left(\mathrm{V}_{1}\right), \mu\left(\mathrm{V}_{2}\right)$ with different spectral weighting. The effective atomic number $\left(\mathrm{Z}_{\text {eff }}\right)$ describes the chemical classification and mass density determines physical morphology of the absorbing material. Both these parameters are used to characterize the material. Although for many substances the $\mathrm{HU}$ values for two different tube voltages can overlap, by applying $(\rho \mathrm{Z})$ projection algorithm they could separate these samples and characterize them (16).

Boll et al. (2006) and Johnson et al. (2007) used dual energy computed tomography ( 90 and $140 \mathrm{kVp}$ ) and subtraction method to enhance the qualitative imaging capabilities. They concluded that spectral dual-energy MDCT imaging of atherosclerotic coronary artery specimens using standard CT scanners will allow tissue characterization. In 2008, they used dual source CT (DSCT) in their studies and concluded that dual-energy MDCT with image post processing techniques enhanced the accuracy of calcified plaque quantification beyond the scope of single-energy MDCT, by using optical coherent tomography (OCT) as the reference standard $(7,17)$.

Further studies are needed to validate CT modalities for the assessment of coronary plaque composition, to provide better differentiation of lipid-rich from fibrous-rich plaques (18).

The present study has the following sections, (1) calibration-inversion of DECT data (2) using the above 
DECT inversion algorithm to determine the $\left(\rho_{e}, \mathrm{Z}_{\text {eff }}\right)$ values of plaque samples, and (3) characterization and discrimination of plaques in terms of their $\left(\rho_{e}, \mathrm{Z}_{\text {eff }}\right)$ values. Although many authors continue to characterize materials on the basis of their HU values, it can give errors, because the HU values depend upon several instrumental factors of the CT machine, including the source spectrum, filtration and detector efficiency.

\section{Objectives}

Our aim in this study was to eliminate these instrument dependent factors. To achieve this, we calibrated the CT machine with respect to $\rho_{e}$ and $Z_{\text {eff }}$, by experimentally determining the $\mathrm{HU}(100)$ and $\mathrm{HU}$ (140) of substances, whose $\rho_{e}$ and $Z_{\text {eff }}$ are known. This calibration gave us the inversion coefficients for determining the $\rho_{e}$ and $\mathrm{Z}_{\mathrm{eff}}$ of unknown substances from their observed HU (100) and HU (140) values. For characterization of non-calcified coronary artery plaque, we used the $\mathrm{HU}(100), \mathrm{HU}(140)$ data and applied the above inversion algorithm to calculate their $\left(\rho_{e}\right.$, $\left.\mathrm{Z}_{\text {eff }}\right)$. These quantities $\left(\rho_{e}, \mathrm{Z}_{\text {eff }}\right)$ were directly related to the chemical characteristics of the plaque material and were thus used as basic inputs for plaque characterization.

\section{Materials and Methods}

\subsection{Ex-Vivo Study}

This study was approved by the ethical committee of the All India Institute of Medical Sciences (AIIMS), New delhi, India. Left coronary artery of 59 subjects were dissected while the cadavers were fresh (the time elapsed from the time of death was under 24 hours). The excised coronary arteries were from 6 females and 53 males with the age ranges between 20 and 70 years. Samples were selected from the left part of the heart containing left coronary ostium, left main coronary artery (LMCA), left anterior descending artery (LAD), and part of the left circumflex (LCX) of the human cadaver. This selection was based on the prevalence of the plaque on the left coronary artery especially in its proximal segment. Coronary artery samples were extracted from the cadavers who had died from a noncardiac event. Samples were kept in $0.9 \%$ normal saline and refrigerated at 4 degrees Celsius. Maximum duration from the extraction of sample from cadaver and scanning time was 4 hours.

Coronary artery samples were removed from saline just prior to scanning. Left coronary artery was washed by injection of normal saline into the left coronary ostium in order to remove the blood from the coronary arterial lumen. Viscous solution of contrast agent (300 mgI / ml Ominpaque) was prepared by mixing methyl-cellulose with the solution iodinated contrast material in distilled water. The concentration of the viscous material was $2 \%$ weight by weight. The CT slices produced by this concentration were accepted by the cardiac radiologist for coronary artery wall evaluation (Figure $1 \mathrm{~A}-\mathrm{C}$ ). The viscous form of the contrast could be easily injected into the coronary artery by inserting the tip of the syringe into the left coronary ostium. Injection was stopped when the leakage of contrast material was seen from the end of the coronary artery branches. Small amounts of contrast, ejected due to leakage were removed very carefully from the end of the coronary artery branches.

Contrast material in a viscous form was retained in the coronary artery during the scanning procedure. Also, it coats the coronary artery wall permitting better visualization of the coronary artery wall.

Each sample was placed on the scanning table to mimic the process of scanning a real patient for coronary CT angiography (CTA). The coronary ostium was placed in the cranial position and the length of the LAD was oriented along the z-axis of the scanner.

Ex-vivo samples were scanned by Dual Source CT SOMATOM Definition Syngo CT 2008G (Siemens AG, Wittelsbacherplazz, DE-80333 Munchen, Germany). PBV protocol was used to scan coronary artery sample. This protocol uses 100 and $140 \mathrm{kVp}, 64 \times 0.6 \mathrm{~mm}$ beam width, pitch of $0.25,0.33$ s rotation time, automatic tube current modulation (typical mAs values were 15), and the data collected at 360 degree was used to reconstruct the CT images. This protocol uses a demo electrocardiogram (ECG) with 60 beats/minute. B26f convolution kernel was used to reconstruct CT images with $0.6 \mathrm{~mm}$ slice thickness. Data collection diameter or scan field of views (SFOV) were $50 \mathrm{~cm}$ in all cases. Matrix size was $512 \times 512$ and MMWP workstation was used to perform DECT post processing on the raw data.

Two cardiac radiologists with more than 3 years of experience were asked to interpret the images independent of each other and they were blinded to the final pathology result.

On our suggestion to select only non-calcified plaques, the cardiac radiologists rejected the ex-vivo CT images when they could identify any trace of calcification in coronary artery plaque images.

Maximum intensity projection (MIP) and multi-planar reconstruction (MPR) were used to evaluate the presence of the non-calcified plaque throughout the coronary artery lumen by the cardiac radiologist. Actually, all cases where non-calcified plaque were found in the proximal part of LAD and bifurcation of LMCA and LCX, non-calcified plaques were seen identically in MIP images and MPR views. Therefore, MIP images were used to determine the 
position of non-calcified plaque for future visual assessment (Figure 1B) in the present study. It has to be mentioned that MIP views were used only to assess the presence of non-calcified plaque. Therefore, we were not interested in any data such as slice thickness of the MIP images as they were unnecessary for the inversion procedure. Also, we reminded ourselves again that we were searching the noncalcified plaque in the proximal segment of the LAD and in the region of LMCA and LCX bifurcation portion. Visual evaluation was used in this study to verify the adequacy of DECT images to detect non-calcified plaque. Cardiac radiologist used true axial images (Figure 1C) that were made on the basis of MIP images to evaluate and characterize the type of non-calcified plaque.

True axial images from the whole length of the noncalcified plaque were used to measure the density or $\mathrm{HU}$ value of the non-calcified plaque at two different energies (100 and $140 \mathrm{kVp}$ ) in each slice. Size of the region of interest (ROI) is a very important factor in our study. The size of the ROI was selected on the basis of the size of the non-calcified plaques. ROI had to be inside the plaque and should not contaminate the neighbouring structures, avoiding partial volume effect. The typical size of the selected ROI was between 1.1 and $5 \mathrm{~mm}^{2}$. CT images that did not allow more than 3 pixels in the ROI were excluded from this study. The mean and standard deviations of the HU values for the circular ROI were measured three times for each slice to enhance its reliability. Each ROI contained minimum 3 pixels to prevent statistical uncertainty.

$\mathrm{HU}$ values of the structures surrounding the noncalcified plaque such as the border of the wall of the coronary artery, the fat surrounding the coronary artery wall, and the contrast material inside the lumen of the coronary artery were measured at 100 and $140 \mathrm{kVp}$ to evaluate the possibilities of the overlapping (cross-reading) between the HU values of these structures in DECT images (Figure 2).

Samples of the coronary artery which were categorized to have non-calcified plaque (by the cardiac radiologist) were cut cross-sectionally and thin tissue sections were obtained from the center part of the plaque (Figure $1 \mathrm{~A}$ ). These samples were preserved in 10\% formalin and sent to the pathology department. Samples were fixed in paraffin, cut into $5 \mu \mathrm{m}$ slices and stained by hematoxyline eosin for histologic evaluation of the plaque.

\subsection{Experimental Study for DECT Calibration}

In this study, chemical compounds with known effective atomic number $\left(Z_{\text {eff }}\right)$ and electron density $\left(\rho_{e}\right)$ were used to calibrate the DECT machine. These calibration samples were prepared by using high-purity samples of water, methanol, and acetic acid. Apart from these pure com- pounds, we also prepared aqueous solutions of glucose, sucrose, glycerol, citric acid, hydrochloric acid, and potassium hydroxide (19) in suitable compositions. These chemical compounds were selected due to similarity of their $Z_{\text {eff }}$ and $\rho_{e}$ values with those of the typical substances that are present in non-calcified plaque. Chemicals were of analytical purity and were purchased from Merck Chemicals Company. The details of the experimental work are described in Ref. (20). These chemical solutions of known composition were filled in test tubes which were placed at the center of a water filled phantom, the test tubes being thus immersed in water. Both the phantom and test tubes were made of Poly Methyl Methacrylate (PMMA).

Different concentrations of known chemical solutions were prepared (in weight/weight \%) by using digital balance (DENVER Instrument MXX 123, Gottingen, Germany) with $0.01 \mathrm{mg}$ accuracy. Temperature measurements during the experiments were done by using a digital thermometer (Nuclear Associates 07 - 402, Hicksville, NY) with $\pm 0.5^{\circ} \mathrm{C}$ accuracy. Temperature of the $\mathrm{CT}$ room during experiments was fixed at $22 \pm 0.5^{\circ} \mathrm{C}$. The standard method, based on the use of specific gravity bottle, was employed to measure the density of all chemical solutions. The error in density measurement was less than $1 \%$.

Test tubes were filled with liquid samples in pure form and as aqueous mixtures and inserted into the water filled phantom. The phantom containing test tubes was next placed on top of the scanning table in such a way that the length of the test tube was aligned along the $\mathrm{Z}$ axis of the scanning table and was perpendicular to the scanning plane Figure 2 in Ref (20). The phantom and all the samples in the test tubes were scanned by Dual-Source CT which would also be used subsequently to scan the ex-vivo coronary artery samples. For calibration, the phantom was scanned at 100 and $140 \mathrm{kVp}$ and axial slices of the chemical compounds were reconstructed by $5 \mathrm{~mm}$ slice thickness. The circular ROI was placed at the center of each test tube, inside the chemical solutions, without contamination with the neighbouring structures. Mean and standard deviation of HU values were recorded for each ROI. The same procedure was repeated three times and average HU values from these three observations were used to enhance the validity of statistical estimations and analysis. For the same reason, care was taken to select a circular ROI containing 104 pixels in all measurements; thus, statistical fluctuations being minimal. The maximum standard deviation for the selected ROIs was 12 . This shows that the maximum error on the mean value of the HU was $0.32 \%$. Thus, from Equation 21 (20) we calculated the error in the mean $\mathrm{HU}$ value to be typically $0.32 \%$ - which is acceptable. 

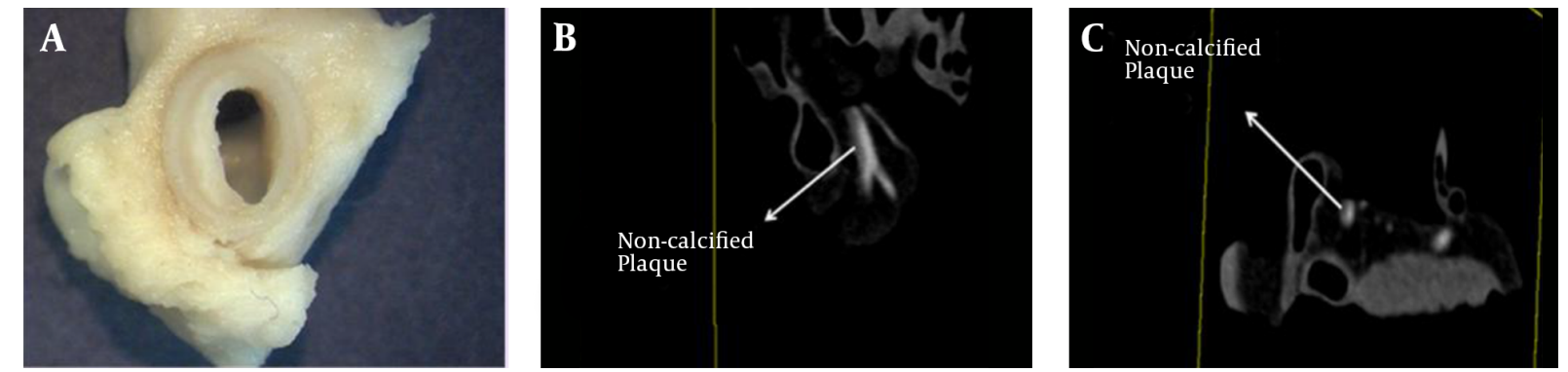

Figure 1. Gross photograph (A), maximum intensity projection (MIP) CT image (B) and axial CT image (C) of non-calcified plaque (shown by arrow adjacent to contrast agent inside the lumen). This sample was collected from a 58-year-old man. Based on the histopathology report, this is a fibro-lipid with a thin fibrous cap, and spotty calcification.

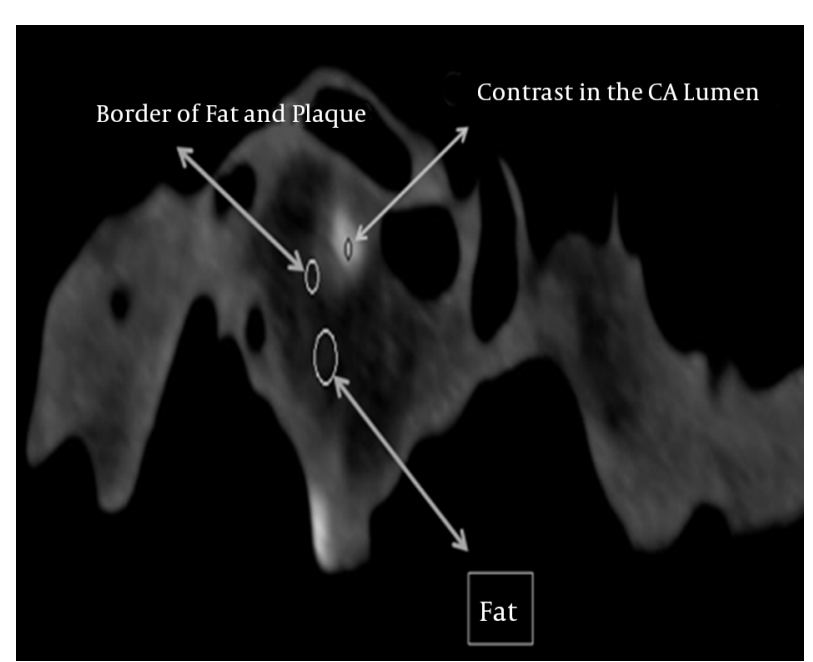

Figure 2. The location of the region of interest (ROI) which are used to measure the Hounsfield unit (HU) value on the fat surrounding coronary artery, the border of fat and wall of the non-calcified plaque, and contrast material in the lumen of coronary artery close to plaque.

\subsection{Theoretical Background for DECT Inversion}

The $\mathrm{HU}\left(\mathrm{V}_{1}\right)$ and $\mathrm{HU}\left(\mathrm{V}_{2}\right)\left(\mathrm{V}_{1}=100\right.$ and $\left.\mathrm{V}_{2}=140 \mathrm{kVp}\right)$ values of the known chemical compounds $\left(\rho_{e}, \mathrm{Z}_{\text {eff }}\right)$ were used to calculate the DECT inversion coefficients. As is known, the $\mathrm{HU}(\mathrm{V})$ of the scanned sample is expressed by Equation (1):

$H U(V)=\frac{\hat{\mu}(V)-\hat{\mu}_{w}(V)}{\hat{\mu}_{w}(V)} \times 1000$

Where $\hat{\mu}(V)$ and $\hat{\mu}_{w}(V)$ are the average linear attenuation coefficients of scanned material and water, respectively. It is known that the linear attenuation coefficient of the scanned material is a function of energy of the x-ray photons, effective atomic number and electron density of the scanned material. Linear attenuation coefficient in the range of $x$-ray energies used in diagnostic radiology (such as CT scanning) is given by Equation (2):
$[\mu(V)]_{\text {Total }}=[\mu(V)]_{\text {Copton }}+[\mu(V)]_{\text {Phtoelectric }}$

The linear attenuation coefficient due to Compton scattering is weakly dependent on the

energy of the x-ray photons, and directly proportional to the electron density of the material.

Photoelectric absorption, on the other hand, depends strongly on the $\mathrm{x}$-ray photon energy (E) and the effective atomic number of the material, the dependence being $\left(\rho_{e}\right.$ $\left.\times\left(Z_{e f f}^{X}\right) / \mathrm{E}^{3}\right)$. Since the $\mathrm{x}$-ray photons produced by $\mathrm{x}$-ray sources are not monoenergetic, the $\mathrm{HU}(\mathrm{V})$ given by the CT machine describes the attenuation coefficient, $\hat{\mu}(V)$, averaged over the source spectrum.

From Equation 1, we can define a quantity $F(V)$ to be in Equations (3) and (4)

$$
\begin{aligned}
F(V) & =\frac{\hat{\mu}(V)}{\hat{\mu}_{w}(V)} \\
& =1+\frac{H U(V)}{1000}
\end{aligned}
$$

and

$F(V)=a(V) \rho_{e}+b(V) \rho_{e} Z_{e f f}^{x}$

where the first term is the contribution from Compton scattering and the second term is from the photoelectric effect. Thus, by taking the ratio of $F\left(V_{1}\right)$ and $F\left(V_{2}\right)$ for two different excitation voltages of the $\mathrm{x}$-ray tube we get,

$f\left(V_{1}, V_{2}\right)=a_{0}+a_{1} Z_{e f f}^{x}$

which depends on $Z_{e f f}^{x}$ alone, $\rho_{e}$ being eliminated, where we have defined $f\left(V_{1}, V_{2}\right)$ by Equation 6 :

$f\left(V_{1}, V_{2}\right)=\frac{F\left(V_{1}\right)}{F\left(V_{2}\right)}$

Thus, if the coefficients $\mathrm{a}_{0}$ and $\mathrm{a}_{1}$ are known, we are able to determine $\left(Z_{\text {eff }}^{x}\right)$ of the substance, from the experimental values of $f\left(V_{1}, V_{2}\right)$, where $f\left(V_{1}, V_{2}\right)$ is calculated from the observed $\mathrm{HU}\left(\mathrm{V}_{1}\right)$ and $\mathrm{HU}\left(\mathrm{V}_{2}\right)$ values of the substance. 
For finding the inversion coefficients, $\mathrm{a}_{0}$ and $\mathrm{a}_{1}$, we take samples with known values of $\left(Z_{\text {eff }}^{x}\right)$ and measure their $\mathrm{HU}\left(\mathrm{V}_{1}\right)$ and $\mathrm{HU}\left(\mathrm{V}_{2}\right)$ with the CT machine that is being calibrated. From these $\mathrm{HU}$ values, $\mathrm{f}\left(\mathrm{V}_{1}, \mathrm{~V}_{2}\right)$ are calculated and fitted to Equation 5. With $\mathrm{a}_{0}, \mathrm{a}_{1}$ determined, Equation 4 serves as the inversion algorithm for $\left(Z_{f f}^{x}\right)$.

From our numerical study of the photoelectric mass attenuation coefficients of substances with different $Z_{\text {eff }}$ values, we found that $Z_{e f f}$ is related to $\left(Z_{e f f}^{x}\right)$, as is shown by Equation 7:

$Z_{\text {eff }}=1.6342\left[Z_{\text {eff }}^{x}\right]^{0.2899}$

where the values of the mass attenuation coefficients were taken from the NIST tables.

The value of $Z_{\text {eff }}$ being known, we have to address next the task of finding the electron density, $\rho_{e}$. On defining, $\mathrm{Y}=\mathrm{F}\left(\mathrm{V}_{2}\right) / \rho_{e}$ and $\mathrm{X}=Z_{\text {eff }}^{x}$ we find that we can write Equation 8:

$Y=c_{0}+c_{1} X$

By making least square fit of the data with Equation 8 for known chemical compounds, the coefficients $\mathrm{c}_{0}$ and $c_{1}$ can be calculated. This method becomes accurate when $\mathrm{HU}$ values of the higher $\mathrm{kVp}$ (for example $140 \mathrm{kVp}$ ) are used, since at those energies photoelectric effect is insignificant and $Z_{e f f}^{x}$ has hardly any contribution to the HU values. Once $c_{0}$ and $c_{1}$ are determined from the data, Equation 8 is the inversion algorithm for finding $\left(\rho_{e}\right)$. In other words,

$\mathrm{HU}\left(\mathrm{V}_{2}\right)$ of unknown materials can be used in Equation 8 to predict $\rho_{e}$ to be,

$\rho_{e}=\frac{F\left(V_{2}\right)}{c_{0}+c_{1} Z_{e f f}^{x}}$

\section{Results}

\subsection{Results of Ex-Vivo Study}

Non-calcified plaque was found in 21 (36\%) ex-vivo coronary artery samples out of 59 . The locations of noncalcified plaques were in the proximal segment of the LAD and at the bifurcation point of the main left coronary artery into LAD and LCX. In DECT, no calcification was detected in any of these 21 cases with pathologic proof of noncalcified plaques (Figures $1 \mathrm{~B}$ and $1 \mathrm{C}$ ). In all cases in which cardiac radiologists reported non-calcified plaque in these regions (in the proximal segment of the $\mathrm{LAD}$ and the bifurcation of LMCA and LCX), macroscopic evaluation could show the presence of non-calcified plaque in the course of coronary artery at the same place.

In DECT images of the ex-vivo samples of non-calcified plaque, discrimination between fibrous, fibro-lipid and lipid core plaques is not possible, the images are the same as MDCT images produced with conventional CT.

CT images have shown that non-calcified plaques have different length and the composition of the plaque is not uniform throughout the span of the plaque. For any particular $\mathrm{kVp}$, the $\mathrm{HU}$ values were significantly different at successive axial slices of each non-calcified plaque (Figure 3A). This was true for both HU (100) and HU (140) in every plaque that we have studied.

It is known that in bone and contrast materials, HU values decrease with the increasing effective energy of the $\mathrm{x}$ ray photons. In these substances $\mathrm{HU}(100)>\mathrm{HU}(140)$. In contrast, for fat, HU values increase with the increasing effective energy of x-ray photons, i.e. for fat $\mathrm{HU}(100)<\mathrm{HU}$ (140). It can be seen in Figure 3A that in each slice of noncalcified plaque $\mathrm{HU}(100)$ can be greater (in most of the cases), very close, or less than HU (140). This finding reconfirms the results published by Okayama et al. (21).

Figure 3B demonstrates the HU values of the structures surrounding non-calcified plaque; namely (a) the wall of the coronary artery, (b) the adipose tissue surrounding the wall of the coronary artery and (c) the contrast material within the lumen of the coronary artery. These areas are completely distinguishable from one another with minimal or no HU cross-reading contamination from their surroundings.

As mentioned earlier, the chemical compounds with a wide range of $Z_{\text {eff }}$ and $\rho_{e}$ (Figure $4 \mathrm{~A}$ ) were used so that they fall within the range of non-calcified plaque compositions. The $\mathrm{Z}_{\mathrm{eff}}$ and $\rho_{e}$ of chemical compounds mimic fat, soft tissue, fibrous tissue and low concentration of contrast materials. Figures 3B and 4B demonstrate that the HU values of non-calcified plaques and the surrounding structures are very similar to the same values (HU values at 100 and $140 \mathrm{kVp}$ ) of chemical compounds that were used in this study. Using these semi-qualitative observations, the $\mathrm{Z}_{\mathrm{eff}}$ and $\rho_{e}$ values were calculated for non-calcified coronary artery plaque, utilizing the inversion procedure. Subsequently, we examined whether these values can be used to characterize the sub-type of non-calcified plaque.

\subsection{Results of DECT Inversion of Non-Calcified Plaque}

HU values of 21 non-calcified plaques at 100 and $140 \mathrm{kVp}$ were measured for all slices through the length of each plaque. Therefore, there are 208 unbiased data points for each HU value used in this study which is quite enough for statistical evaluation and accuracy. While the $\mathrm{HU}(100)$ and HU (140) values of non-calcified plaques are different for each slice inside the plaque, the HU values do not give any information regarding the nature of the plaque by itself (Figure 5). 
A

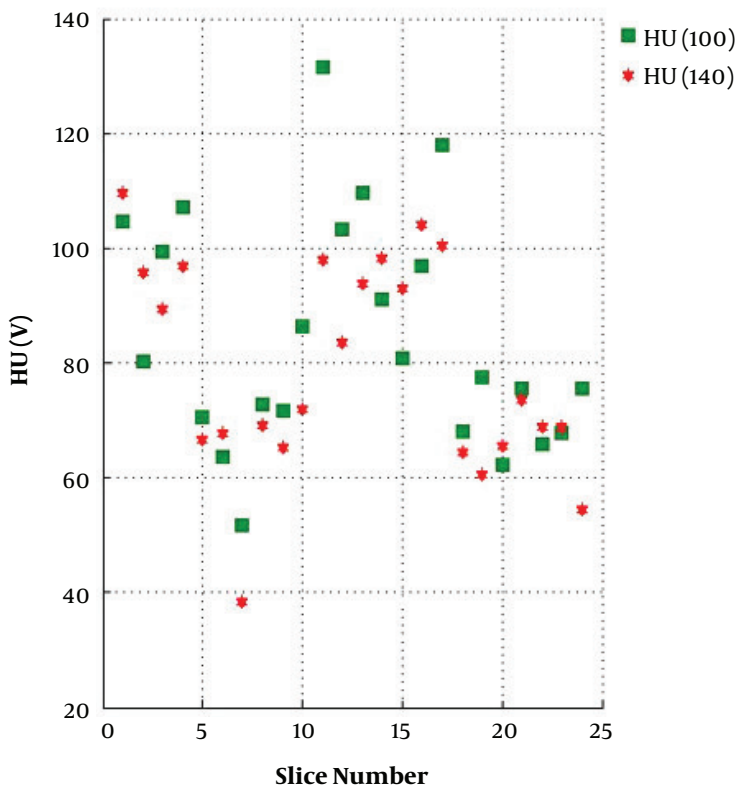

B

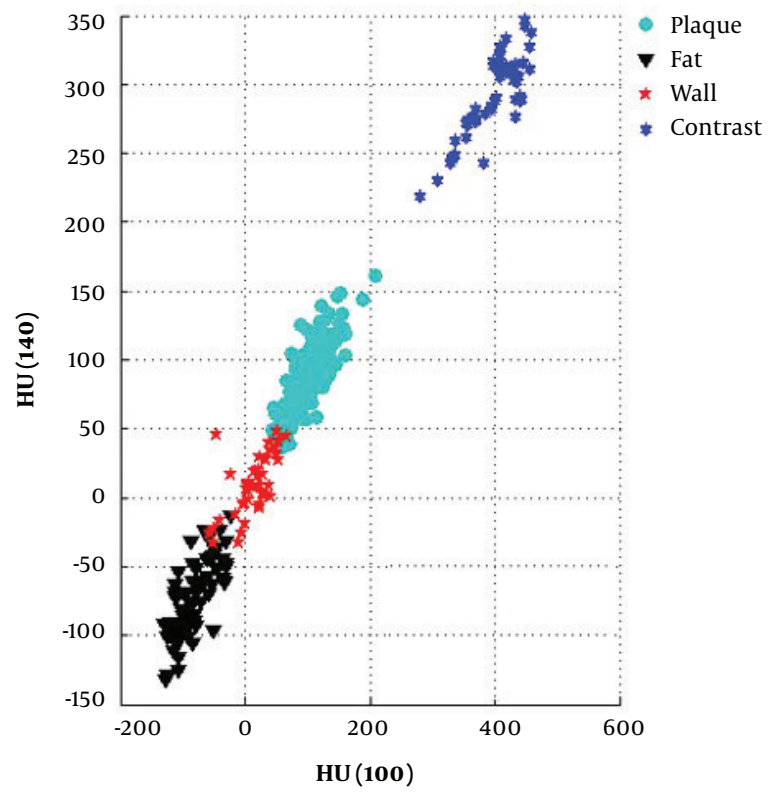

Figure 3. $\mathrm{HU}(100)$ and $\mathrm{HU}(140)$ for each slice in true axial CT images measured through the whole length of non-calcified plaque (A) and neighbouring structures of the noncalcified plaque such as fat surrounding the coronary artery (B), border of fat and wall of the coronary artery, non-calcified plaque and contrast material inside the lumen of the coronary artery (19)

The coefficients of inversion, found by calibration of the CT machine, were used in the inversion algorithms to convert the $\mathrm{HU}(100)$ and $\mathrm{HU}(140)$ values into the $Z_{\text {eff }}$ and $\rho_{e}$. Figure 6 demonstrates the scatter diagram of the result of inversion of these $\mathrm{HU}$ values for all the 208 data points. Every data point in the scatter diagram shows the $Z_{\text {eff }}$ and $\rho_{e}$ value for a given slice in the non-calcified plaque samples.

It is known that plaque consists of (a) fibres, (b) lipid, and (c) calcium phosphate. We assume that these constituents are present in weight/weight\% proportion $\mathrm{w}_{1}: \mathrm{w}_{2}$ : $\mathrm{w}_{3}$, respectively.

Further, the typical $\left(\rho_{e}, Z_{\text {eff }}\right)$ values for lipids, fibers and calcium phosphate are $\left(5.5,3.0 \times 10^{23} / \mathrm{cm}^{3}\right),(6.5,4.4 \times$ $\left.10^{23} / \mathrm{cm}^{3}\right)$ and $\left(19,9.4 \times 10^{23} / \mathrm{cm}^{3}\right)$, respectively. But our DECT inversion results (Figure 6$)$ show that $\left(\rho_{e}, Z_{\text {eff }}\right)$ of noncalcified plaque are higher than those for pure fibrous and lipid materials. It can be attributed to very small amount of calcium phosphate, present in the plaques. Histopathology study also reported micro-calcification (about $2 \mu \mathrm{m}$ in size) in the lipid part of the non-calcified plaques.

Elemental analysis of the results of DECT inversion for $\left(\rho_{e}, Z_{\text {eff }}\right)$ shows that plaque material can have different proportions of lipid $\left(\mathrm{w}_{1}\right)$, fibrous material $\left(\mathrm{w}_{2}\right)$ and calcium phosphate $\left(w_{3}\right)$. The scatter diagram in Figure 6 shows that the $\left(\rho_{e}, Z_{\text {eff }}\right)$ points in the scatter diagram lie in four distinct regions. We now intend to correlate the locations of these points with the composition of the plaque. The values of the effective atomic number and electron density $\left(\rho_{e}, \mathrm{Z}_{\mathrm{eff}}\right)$ are to serve as the parameters for identification of the nature of the coronary artery plaque. Since elemental compositions of these substances are not exactly known (known in a broad sense though) one has to depend on the known qualitative features about these substances.

In Figure 6 the material whose $\left(\rho_{e}, Z_{\text {eff }}\right)$ values lie in quadrant $\mathrm{A}$, can be clearly identified to be fibrous plaque. Fibers are made of a mixture of proteins, water and other minerals. Owing to the presence of a considerable amount of water in proteins (molecular basis of the fiber) the fibrous and lipid parts remain segregated, due to immiscibility of water and lipids. Also, with the increasing fibrous content, the proportion of lipid and calcium decreases.

The modeling for the ones in which $\left(\rho_{e}, \mathrm{Z}_{\text {eff }}\right)$ lie in quadrants $B$ and $C$ is difficult. It is known that most lipids, e.g., fats and triglycerides in their pure form have density lying in the range, $0.9-0.96 \mathrm{gm} / \mathrm{cc}$. Since lipids have a tendency to acquire micro-calcification, their electron densities in the plaque may be higher than those for pure lipids and the values of the effective atomic numbers may be considerably higher. We found that as the concentration of lipid increases, the concentration of calcium phosphate also increases in the plaque. Calcium phosphate is seen to be 
A

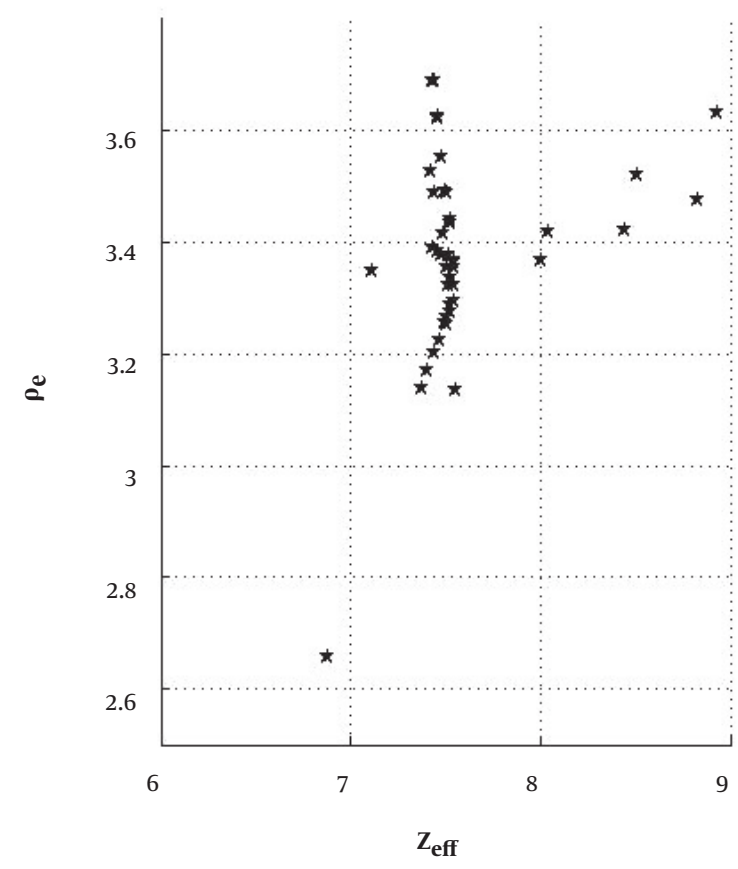

B

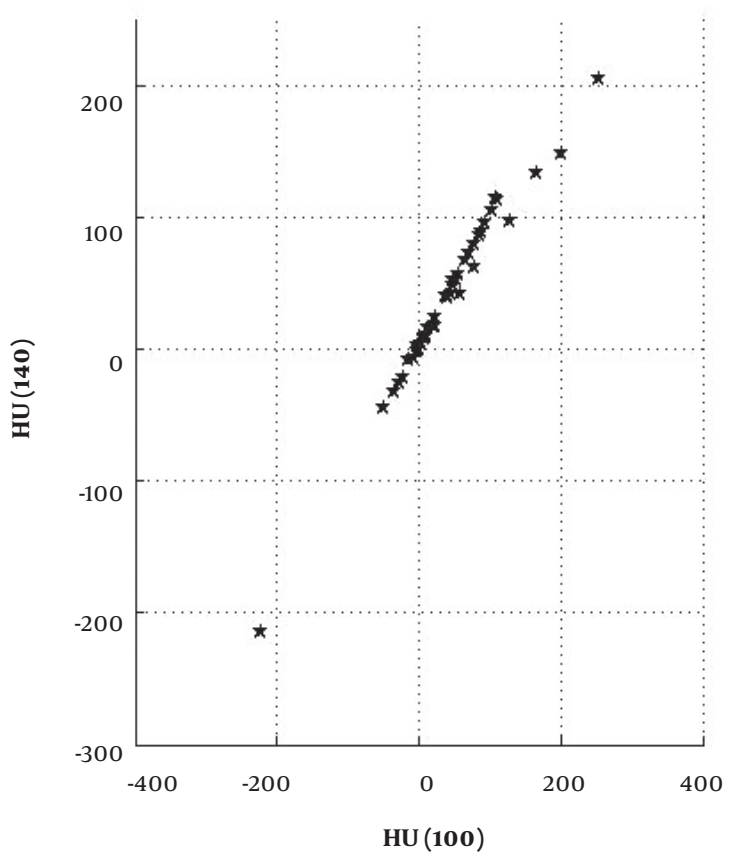

Figure 4. Scatter diagram of $\mathrm{A}, \mathrm{Z}_{\mathrm{eff}}$ and $\rho_{e 10^{23}}$ and $\mathrm{B}, \mathrm{HU}(100)$ versus $\mathrm{HU}(140)$ of the chemical compound used for calibration inversion purposes (19)

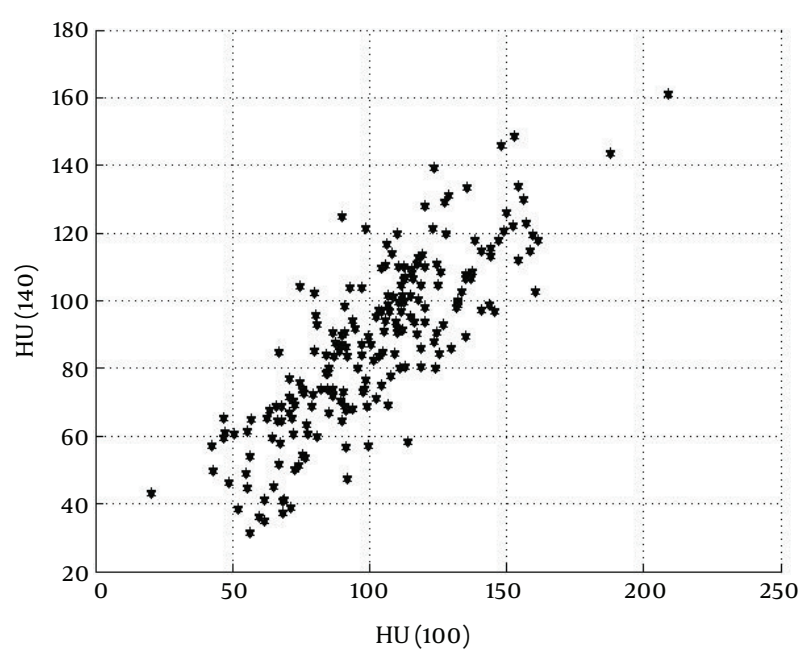

Figure 5. Scatter diagram of HU (100) versus HU (140) of 208 data points of noncalcified coronary artery plaques belonging to 21 human heart cadaver samples

present in the lipid-rich part of the plaque. This was also reported by van der Berg et al. (22). The parts in quadrants $B$ and $C$ represent fibro-lipid parts with calcification and are modeled in terms of varying compositions of proteins, lipids and calcium. We can conclude that data points in

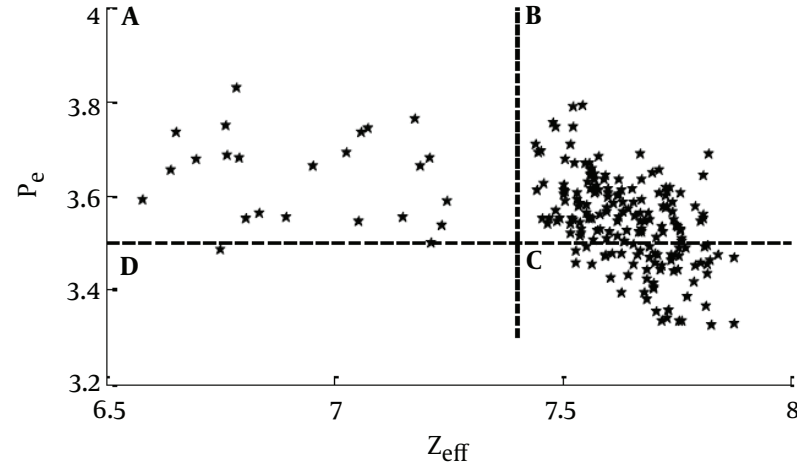

Figure 6. $Z_{\text {eff }}$ versus $\rho_{e 10^{23}}$ of non-calcified coronary artery plaque from $208 \mathrm{sec}$ tions. Region A: represents the fibrous part; Region B: represents fibrous plaque plus lipid with calcification; Region C: corresponds to lipid with calcification with hardly any fibrous parts (thin fibrous cap); and Region D: there is no sample

quadrant $C$ of Figure 6 have less fiber, as compared to those in quadrant B. As we note, there is no sample of those $\left(\rho_{e}\right.$, $\mathrm{Z}_{\text {eff }}$ ) points that lie in quadrant D of Figure 6.

Therefore, based on the above elemental model, we conclude that:

(a) In ex-vivo samples diagnosed as lipid-rich, the large number of slices showed that $\mathrm{w}_{2}>0.5$, i.e. lipid content 
was more than $50 \%$ by weight;

(b) Similarly, in samples that were found as fibrous plaque, we found that there were large portions with $\mathrm{w}_{1}>$ 0.5 ;

(c) In plaques diagnosed with thin fibrous cap, there were large parts with $\mathrm{w}_{1}<0.15$, and several sections with $\mathrm{w}_{1}<0.3$, i.e. presence of fiber was found to be low;

(d) In samples without visible (either in CT image or in histopathology) micro-calcification, there were sections with $\mathrm{w}_{3}<0.03$, i.e. with an extremely low calcium content.

Thus, with DECT inversion we can get semiquantitative estimates of $\mathrm{w}_{1}, \mathrm{w}_{2}$, and $\mathrm{w}_{3}$. These estimates can enable us to determine a method by which we can distinguish between different types of non-calcified plaques like (a) fibrous (region A of Figure 6), (b) fibro-lipid (region B of Figure 6), and (c) lipid rich with thin fibrous cap (region $C$ of Figure 6) plaques. It has to be noted that fibro-lipid and lipid rich plaques contain microcalcification. Classifications based on histopathology study corroborated our finding in $63 \%$ of the cases.

\section{Discussion}

Physical techniques are becoming more and more important as diagnostic tools for physicians, particularly since about a hundred and twenty years ago when x-rays were discovered. The most recent application of $x$-rays is in the Dual Energy Computed Tomography (DECT) for chemical characterization of unseen objects, for non-invasive diagnostics. In this paper, we have tried to examine this possibility with DECT for characterization of coronary artery plaque, a method that can prove to be an asset for cardiologist and radiologist alike, the ultimate beneficiary being the patient.

This study showed that DECT inversion is able to convert $\mathrm{HU}\left(\mathrm{V}_{1}\right)$ and $\mathrm{HU}\left(\mathrm{V}_{2}\right)$ of the scanned slice into $\rho_{e}$ and $\mathrm{Z}_{\text {eff }}$ of the scanned sample. Also, it is shown that from the results of our DECT inversion algorithm we can distinguish the non-calcified plaque to be (a) fibrous, (b) fibro-lipid (c) lipid type with significant calcium contents. Thus, while the CT image of non-calcified plaque cannot discriminate lipid and fibrous plaques and also is not able to show micro-calcification inside the lipid plaques, DECT inversion can make these distinctions on the basis of the calculated $\mathrm{Z}_{\mathrm{eff}}$ and $\rho_{e}$ values. Also, from qualitative analysis of $\mathrm{Z}_{\mathrm{eff}}$ and $\rho_{e}$ it was concluded that non-calcified plaques containing lipid have micro-calcification which cannot be seen in CT images but can be corroborated with histopathology studies. A. Vedre et.al. have also stated that cholesterol aggregates can be found around the calcium part and have studied the evolutionary process of aggregate formation (23). Molecular structure of cholesterol shows that it has polar head and a non-polar tail, where its polar head is small but the non-polar part is longer. Therefore, cholesterol does not dissolve in water and the non-polar part of cholesterol attracts large number of lipid and fatty acids. As a consequence, it is natural that microcalcification is present in non-calcified plaque in lipid part of the plaque $(23,24)$.

It should be stated that histopathology study with light-microscopy evaluation is able to reveal the microcalcifications only when the size of micro-calcification is greater than $2 \mu \mathrm{m}$. As shown by the present study, DECT inversion can detect the presence of even smaller quantities of calcium. What is even more promising is that although, micro-calcifications of size less than the spatial resolution of the CT system cannot be seen in the CT image, it has significant effects on HU values (shift the HU values to the higher side). Thus, DECT inversion is able to demonstrate the existence of micro-calcifications which could not be seen by light-microscopy or in CT images.

The importance of the DECT inversion developed in this study is that the results are independent of the characteristics of the CT machine. We are able to extract important chemical parameters, like $\rho_{e}$ and $\mathrm{Z}_{\text {eff }}$ of the material. This is achieved by calibrating the CT machine with known samples. This calibration-inversion algorithm can be used to determine the $\left(\rho_{e}\right.$ and $\left.Z_{\text {eff }}\right)$ values of any unknown material.

In summary, the present work combines several steps. Firstly, we have standardized a method for CT scanning of ex-vivo coronary artery, by injecting contrast material in viscous form, a method that was first used by Becker et.al. (25). By this, we have developed a method that allows enough time to scan ex-vivo samples without any risk of losing contrast enhancement in the quality of the image, as was checked by radiologists.

Next, from this study we could develop a new method to determine chemical composition $\left(\rho_{e}, \mathrm{Z}_{\mathrm{eff}}\right)$ of noncalcified plaque by using DECT inversion. All factors regarding source spectrum or detector efficiency are eliminated by this single stroke of calibration $(19,26)$. This can also be checked by model calculations based on mass attenuation table (27). Pixel by pixel tissue decomposition by this method is also possible and would be developed as a very useful method to determine the vulnerability of coronary artery plaque. It will help physicians to take proper decision and on time treatment of CAD patients.

This study is our first attempt to do experimental work with ex-vivo coronary artery. It has to be repeated in several cases and then linked to the study with living patients, once its repeatability and reproducibility are established.

For this, we are in the process of setting up experimental phantoms that closely represent the human heart. That 
will provide further advance to our aim of characterization of coronary artery plaque through diagnostic medical imaging by DECT inversion.

\section{Acknowledgments}

Dr.(Ms.) Rezvan Ravanfar Haghighi thanks Shiraz University of Medical Sciences for granting her leave and the all India Institute of Medical Sciences, New Delhi, India for providing the facilities. The authors acknowledge the financial support received from Indian Council of Medical Research (ICMR), New Delhi through Project No. 5/20/8(Bio)/09-NCD-I.

\section{Footnotes}

Author's Contributions: Rezvan Ravanfar Haghighi and Sabyasachi Chatterjee, study concept and design; Rezvan Ravanfar Haghighi, data acquisition; Rezvan Ravanfar Haghighi, Sabyasachi Chatterjee and Vardahan.C. Vani, analysis and interpretation of data; Rezvan Ravanfar Haghighi, Reza Jalli, Sepide Sefidbakht, Fariba Zarei, Ali Reza Shakibafard, Somayeh Gholami Bardeji and Mahdi Dodangeh, intellectual input; Rezvan Ravanfar Haghighi, Sabyasachi Chatterjee and Bijan Bijan, critical revision of the manuscript for important intellectual content; Rezvan Ravanfar Haghighi and Sabyasachi Chatterjee, statistical analysis; Pratik Kumar, Milo Tabin, Ruma Ray, Reza Jalli and Sepide Sefidbakht, administerative, technical and material support; Sabyasachi Chatterjee, Study supervision.

Funding / Support: Role of the Sponsor: The funding organizations were governmental institutions and approved the study. The medical physics unit, cardiac radiology, biochemistry, pathology, and forensic medicine departments of the All India Institute of Medical Sciences provided practical support to collect samples, make chemical solutions and for doing the DECT experiments. Shiraz University of Medical sciences granted the leave for the first author to do the study. The respective institutes sponsored this research. Funding is in the form of salary.

\section{References}

1. Adiguzel E, Ahmad PJ, Franco C, Bendeck MP. Collagens in the progression and complications of atherosclerosis.Vasc Med.2009;14(1):73-89. doi: 10.1177/1358863X08094801. [PubMed: 19144782].

2. Greenland P, Smith SJ, Grundy SM. Improving coronary heart disease risk assessment in asymptomatic people: role of traditional risk factors and noninvasive cardiovascular tests. Circulation. 2001;104(15):1863-7. [PubMed: 11591627].

3. Fuster V, Fayad ZA, Badimon JJ. Acute coronary syndromes. Biolog Lancet. 1999;353:s5-9.
4. Nair A, Kuban BD, Tuzcu EM, Schoenhagen P, Nissen SE, Vince DG. Coronary plaque classification with intravascular ultrasound radiofrequency data analysis. Circulation. 2002;106(17):2200-6. [PubMed: 12390948].

5. Kume T, Akasaka T, Kawamoto T, Watanabe N, Toyota E, Neishi $\mathrm{Y}$, et al. Assessment of coronary arterial plaque by optical coherence tomography. Am J Cardiol. 2006;97(8):1172-5. doi: 10.1016/j.amjcard.2005.11.035. [PubMed: 16616021].

6. Vaina S, Stefanadis C. Detection of the vulnerable coronary atheromatous plaque. Where are we now? Int J Cardiovasc Intervent. 2005;7(2):75-87. doi: 10.1080/14628840510011252. [PubMed: 16093216].

7. Boll DT, Hoffmann MH, Huber N, Bossert AS, Aschoff AJ, Fleiter TR. Spectral coronary multidetector computed tomography angiography: dual benefit by facilitating plaque characterization and enhancing lumen depiction. J Comput Assist Tomogr. 2006;30(5):804-11. doi: 10.1097/01.rct.0000228162.70849.26. [PubMed: 16954934].

8. Horiguchi J, Fujioka C, Kiguchi M, Shen Y, Althoff CE, Yamamoto H, et al.Soft and intermediate plaques in coronary arteries: how accurately can we measure CT attenuation using 64-MDCT? AJR Am J Roentgenol. 2007;189(4):981-8. doi: 10.2214/AJR.07.2296. [PubMed:17885074].

9. Kramer CM, Anderson JD. MRI of atherosclerosis: diagnosis and monitoring therapy. Expert Rev Cardiovasc Ther. 2007;5(1):69-80. doi: 10.1586/14779072.5.1.69. [PubMed: 17187458].

10. Schnapauff D, Zimmermann E, Dewey M. Technical and clinical aspects of coronary computed tomography angiography. Semin Ultrasound CT MRI. 2008;29(3):167-75.

11. Schneider W, Bortfeld T, Schlegel W. Correlation between CT numbers and tissue parameters needed for Monte Carlo simulations of clinical dose distributions. Physic Med Biolog. 2000;45(2):459-78.

12. Cademartiri F, La Grutta L, Palumbo AA, Maffei E, Runza G, Bartolotta TV, et al. Coronary plaque imaging with multislice computed tomography: technique and clinical applications. Europ Radiol Suppl. 2006;16(7):M44-53.

13. Macovski A, Alvarez RE, Chan JLH, Stonestrom JP, Zatz LM. Energy dependent reconstruction in X-ray computerized tomography. Comput Biol Med.1976;6(4):325IN7335-334336.

14. Rutherford RA, Pullan BR, Isherwood I. Measurement of effective atomic number and electron density using an EMI scanner. Neuroradiology. 1976;11(1):15-21. [PubMed: 934468].

15. Chiro GD, Brooks RA, Kessler RM, Johnston GS, Jones AE, Herdt JR, et al. Tissue signatures with dual-energy computed tomography. Radiology.1979;131(2):521-3. doi: 10.1148/131.2.521. [PubMed: 441344].

16. Heismann BJ, Leppert J, Stierstorfer K. Density and atomic number measurements with spectral x-ray attenuation method. J Appl Physic. 2003;94(3):2073-9.

17. Johnson TR, Krauss B, Sedlmair M, Grasruck M, Bruder H, Morhard D, et al. Material differentiation by dual energy CT: initial experience. Eur Radiol. 2007;17(6):1510-7. doi: 10.1007/s00330-006-0517-6. [PubMed: 17151859].

18. Tanami Y, Ikeda E, Jinzaki M, Satoh K, Nishiwaki Y, Yamada M, et al. Computed tomographic attenuation value of coronary atherosclerotic plaques with different tube voltage: an ex vivo study. J ComputAssist Tomogr.2010;34(1):58-63. doi:10.1097/RCT.0b013e3181b66c41. [PubMed: 20118723].

19. Haghighi RR, Chatterjee S, Tabin M, Sharma S, Jagia P, Ray R, et al. DECT evaluation of noncalcified coronary artery plaque. Med Phys. 2015;42(10):5945-54. doi: 10.1118/1.4929935. [PubMed: 26429269].

20. Haghighi RR, Chatterjee S, Vyas A, Kumar P, Thulkar S. X-ray attenuation coefficient of mixtures: inputs for dual-energy CT. Med Phys. 2011;38(10):5270-9. doi: 10.1118/1.3626572. [PubMed: 21992344].

21. Okayama S, Soeda T, Takami Y, Kawakami R, Somekawa S, Uemura S, et al. The influence of effective energy on computed tomography number depends on tissue characteristics in monoenergetic cardiac imaging. Radiol Res Pract. 2012;2012.

22. Van den Berg AA, Van Buul JD, Tytgat GNJ, Groen AK, Ostrow JD. Mucins and calcium phosphate precipitates additively stimulate cholesterol crystallization. J Lipid Res. 1998;39(9):1744-51. 
23. Vedre A, Pathak DR, Crimp M, Lum C, Koochesfahani M, Abela GS. Physical factors that trigger cholesterol crystallization leading to plaque rupture. Atherosclerosis. 2009;203(1):89-96. doi: 10.1016/j.atherosclerosis.2008.06.027. [PubMed:18703195].

24. Srinivasan A, Ramaswamy V, Kuruvilla S, Sehgal PK, Balakrishnan K. Calcified atherosclerotic plaque-where exactly is the calcium and what does it contain? India J Thorac Cardiovasc Surg. 2012;28(1):6-14.

25. Becker CR, Nikolaou K, Muders M, Babaryka G, Crispin A, Schoepf U, et al. Ex vivo coronary atherosclerotic plaque characteriza- tion with multi-detector-row CT. Eur Radiol. 2003;13(9):2094-8. doi: 10.1007/s00330-003-1889-5. [PubMed: 12692681].

26. Haghighi RR, Chatterjee S, Kumar P, Chatterjee V. Numerical Analysis of the Relationship between the Photoelectric Effect and Energy of the X-Ray Photons in CT. Front Biomed Technol. 2015;1(4):240-51.

27. Hubbell $\mathrm{JH}$, Seltzer SM. Table of X-ray mass attenuation coefficients from 1 keV to $20 \mathrm{MeV}$ for elements $Z=1$ to 92 and 48 additional substances of dosimetric interest. 05 Apr 2017. Available from: http://physics.nist.gov/ PhysRefData/Xcom/html/xcom1.html. 